\title{
Parallax-Free Long Bone X-ray Image Stitching
}

\author{
Lejing Wang ${ }^{1}$, Joerg Traub ${ }^{1}$, Simon Weidert ${ }^{2}$, Sandro Michael Heining ${ }^{2}$, \\ Ekkehard Euler ${ }^{2}$, and Nassir Navab ${ }^{1}$ \\ 1 Chair for Computer Aided Medical Procedures (CAMP), TU Munich, Germany \\ 2 Trauma Surgery Department, Klinikum Innenstadt, LMU Munich, Germany
}

\begin{abstract}
In this paper, we present a novel method to create parallaxfree panoramic X-ray images of long bones during surgery by making the $\mathrm{C}$-arm rotate around its X-ray source, relative to the patient's table. In order to ensure that the $\mathrm{C}$-arm motion is a relative pure rotation around its X-ray source, we move the table to compensate for the translational part of the motion based on C-arm pose estimation, for which we employed a Camera Augmented Mobile C-arm system [1] and a visual planar marker pattern. Thus, we are able to produce a parallax-free panoramic $\mathrm{X}$-ray image that preserves the property of linear perspective projection. We additionally implement a method to reduce the error caused by varying intrinsic parameters of C-arm X-ray imaging. The results show that our proposed method can generate a parallax-free panoramic X-ray image, independent of the configuration of bone structures and without the requirement of a fronto-parallel setup or any overlap in the X-ray images. The resulting panoramic images have a negligible difference (below 2 pixels) in the overlap between two consecutive individual X-ray images and have a high visual quality, which promises suitability for intra-operative clinical applications in orthopedic and trauma surgery.
\end{abstract}

\section{Introduction and Related Works}

Orthopedic and trauma surgery rely heavily on intra-operative X-ray images to visualize bone fracture configurations and to support repositioning and osteosynthesis, especially in minimally invasive surgery. Mobile C-arms are an everyday tool for acquiring X-ray images in the operating room during surgery. However, $\mathrm{X}$-ray images acquired by mobile $\mathrm{C}$-arms have a narrow field of view and often can not visualize the entire bone structure of interest within a single X-ray image, especially in long bone fixation surgery. Determining the relative position and orientation of bone segments across several individual images will definitely result in a compromise of accuracy and may make the surgery more complex. Panoramic X-ray images with exceptionally wide fields of view could be very helpful in these cases, since they can show the whole bone structure in a single image with a large field of view.

Panoramic X-ray images can be obtained by stitching many individual X-ray images. For intra-operative X-ray stitching one method was proposed by Yaniv and Joskowicz 2] using a standard mobile C-arm. This method introduces an orthopedic radiolucent X-ray ruler placed along and parallel to the bones. It 
uses the graduations of the ruler in the images to estimate the planar transformation by a feature-based alignment method and requires the user to manually select the reconstruction plane in order to compensate for parallax effects on that plane. However, this method requires overlapping areas between two consecutive $\mathrm{X}$-ray images to estimate the planar transformation and thus needs additional radiation. Furthermore, it requires a fronto-parallel $\mathrm{C}$-arm setup, i.e. the ruler plane must be parallel to the detector plane of the C-arm. Wang et al. [3] presented a method to generate panoramic X-ray images intra-operatively using a $\mathrm{C}$-arm, to which a video camera was attached. This method does not require overlapping X-ray images or special X-ray markers. It uses the video images in combination with a visual marker pattern to estimate the planar transformation for creating panoramic X-ray images. In order to reduce the parallax effects on the bone plane, the marker plane and the bone plane need to be parallel to each other and the distance between them has to be manually estimated.

Parallax effects will cause ghosting and misalignment in the panoramas (see figure 1(a) . Although methods for reducing parallax effects on the bone plane were described in [23], they require the bone segments to be on the same plane and parallel to the plane used for estimating the transformation. In practice, it is unlikely that the plane of the bone is exactly parallel to the plane of the ruler (or marker pattern) due to the soft tissue around bones. Bone segments furthermore may be on different plane levels due to fracture or inability to fully extend the joints. For these complex situations, completely removing the parallax effects in the panoramic images is impossible by using the early introduced methods [2]3. This will finally restrict the stitching approaches within clinical routine to a limited number of cases.

The pure rotation of the mobile $\mathrm{C}$-arm around its X-ray source is a basic requirement for stitching individual X-ray images to a true parallax-free panoramic $\mathrm{X}$-ray image, which preserves the linear perspective projection. However, making the mobile $\mathrm{C}$-arm rotate around its X-ray source is impractical or even impossible, due to the limitation of the mechanical configuration of mobile C-arms. In this paper, we propose a new method for parallax-free X-ray image stitching by making the $\mathrm{C}$-arm rotate around its X-ray source, relative to the patient's table. This is achieved by moving the table to compensate for the translational part of the motion based on C-arm pose estimation, for which we employed a Camera Augmented Mobile C-arm (CamC) system and a visual planar marker pattern. This CamC system that extends a standard mobile C-arm by a video camera and mirror construction was first proposed for X-ray and video image overlay [1]. Finally, we are able to generate a true parallax-free panoramic Xray image, independent of the configuration of bone structures and without the requirement of a fronto-parallel setup or overlapping $\mathrm{X}$-ray regions. This true panoramic X-ray image now can be treated as a single image obtained by a Carm having an exceptional wide imaging field of view and also can be further processed by various computer vision algorithms that assume linear perspective projection. 

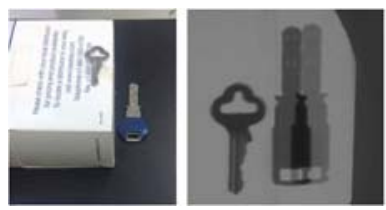

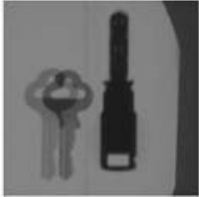

(a)
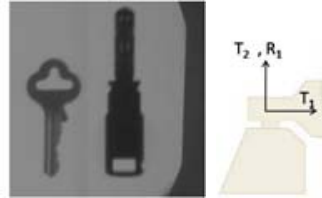

(b)

Fig. 1. (a) Illustration of the parallax effects in the panorama of stitching two X-ray images. From the left to right, the first image shows the construction of two metal keys on two different parallel planes. The second image is the panorama generated using the transformation for the plane of the smaller key, and the third image shows the panorama generated using the transformation for the plane of the bigger key. The last image presents a parallax-free panorama generated by the X-ray source undergoing a relative pure rotation; (b) Available movements for C-arms. 3 rotations around 3 orthogonal axes $R_{1}, R_{2}$ and $R_{3}, 2$ translations along 2 orthogonal axes $T_{1}$ and $T_{2}$.

\section{System Setup}

Our system is composed of a planar square marker pattern, a translatable operating table and a CamC system built by attaching a video camera and mirror construction to a mobile C-arm. Our designed visual square marker pattern (see figure 2(a) was printed in A2 size paper by a high definition printer. This marker

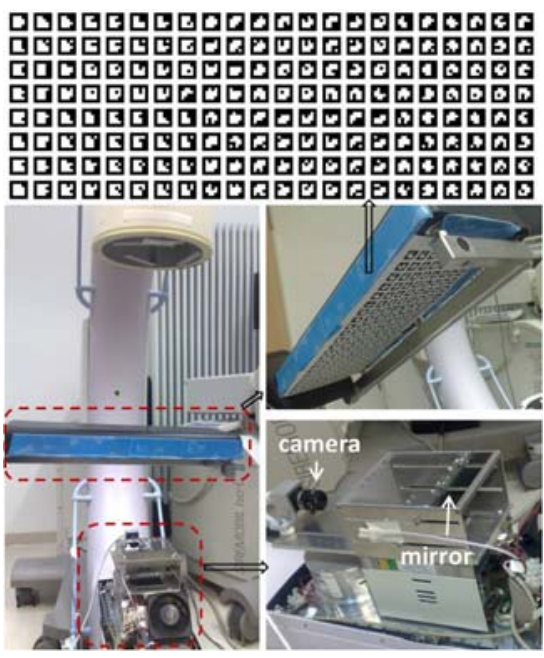

(a) System setup

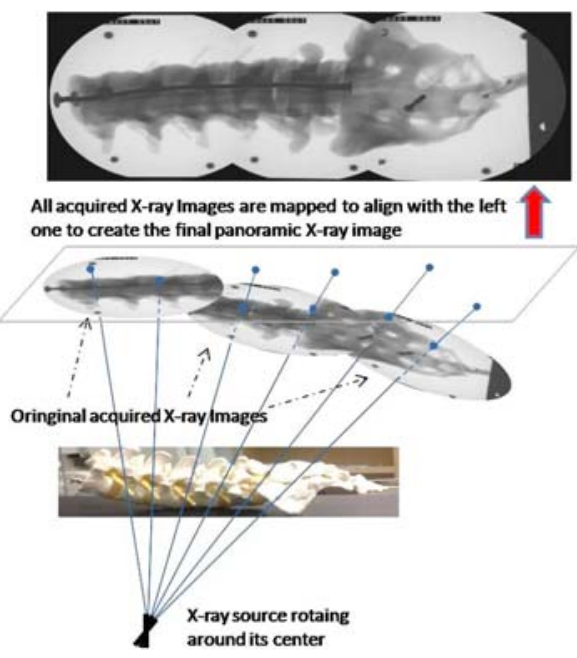

(b) Parallax-free stitching

Fig. 2. (a) The top image shows our square marker pattern. The bottom left image shows the setup for X-ray image stitching. The bottom right image shows the attachment of the marker pattern to the table back and our custom made camera and mirror construction; (b) A parallax-free panoramic image of a plastic lumbar spine and sacrum is generated by having the X-ray source rotate around its center relative to the object. 


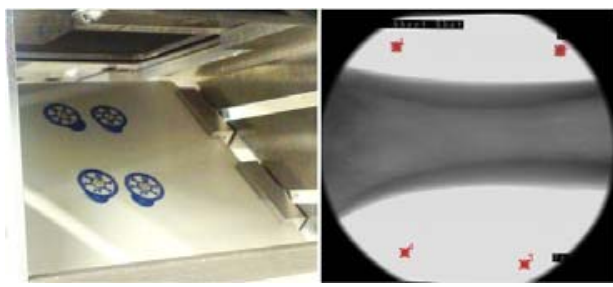

(a) VDP implementation

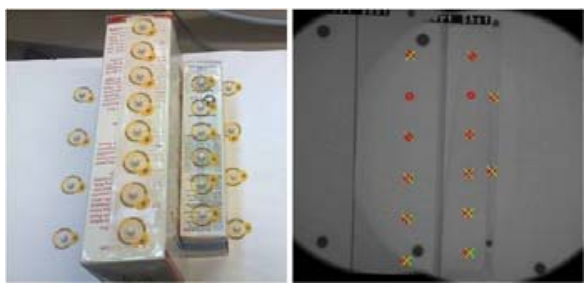

(b) Parallax-free Stitching Quantification

Fig. 3. (a) The left image shows four X-ray markers attached on the back of the mirror. The right image shows the extracted centroids of the X-ray markers in the X-ray image marked by red crosses with indices; (b) The left image shows a phantom of spherical $\mathrm{X}$-ray markers on three different planes. The right image shows that the extracted centroids of the X-ray markers are marked by red and green crosses in the two X-ray images stitched by using our method.

pattern was rigidly and flatly attached under the operating table. The mobile $\mathrm{C}$-arm will be positioned in the common setup, in which the X-ray source is below the operating table (see figure 2(a) . In the method proposed by Wang et al. 3], the marker pattern was placed above the patient and could occlude the view onto the operation situs. Compared to their solution, our system setup does not only make the marker pattern visible to the camera for pose estimation, but also let the marker pattern be smoothly integrated into the surgical procedure without disturbing the physician's view. In order to reduce the error caused by varying intrinsic parameters of C-arm X-ray imaging, we attached four spherical $\mathrm{X}$-ray markers with $1.5 \mathrm{~mm}$ diameter on the back of the mirror, near the border of and within the X-ray's field of view (see figure $3(\mathrm{a})$.

\section{Method}

The camera is attached to the C-arm such that its optical center virtually coincides with the X-ray source. A planar homography then is estimated for X-ray and video image overlay. This calibration enables that the X-ray source and the video camera have the same intrinsic and extrinsic parameters [1]. Therefore, all the poses and motions estimated using the video camera directly correspond to that of the X-ray projection geometry.

The key step of image stitching is the estimation of the planar homography to align images. The camera pose of acquiring the first image is defined as the relative world coordinate system, and the second image is obtained after a rotation $R \in \mathbb{R}^{3 \times 3}$ and a translation $t \in \mathbb{R}^{3}$ of the camera. The planar homography that aligns the first camera image to the second camera image is defined by $H=K R K^{-1}+\frac{1}{d} K \operatorname{tn}^{T} K^{-1}$ where $K \in \mathbb{R}^{3 \times 3}$ is the intrinsic matrix of the camera. $H$ is valid for all image points whose corresponding space points are on the same plane, called stitching plane, defined by the normal vector $n \in \mathbb{R}^{3}$ and distance $d$ to the origin in the world coordinate system [2]. However, any structure 
that is not within this stitching plane in $3 \mathrm{D}$ space will get ghosting or blurring effects (see figure 1(a) also refered to as parallax. $H$ has two parts, $K R K^{-1}$ and $\frac{1}{d} K t n^{T} K^{-1}$, in which the second part relies on the plane parameters $n$ and $d$. In case that the camera motion only contains the rotation $R, \frac{1}{d} K t n^{T} K^{-1}$ is equal to zero. Then $H$ is independent from the plane parameters, and thus it is valid for all image points without the coplanar constraint on their corresponding space points.

Parallax-Free X-ray Stitching. In real clinical cases, it is hard to assume that the bone structure is a simple and planar structure. Rotating the mobile C-arm around its X-ray source is an indispensable step for generating a panoramic Xray image without any parallax effect. However, it is difficult and even impossible to make a pure rotation around the X-ray source, because standard mobile Carms have only 5DOF (3 rotations around axes $R_{1}, R_{2}$ and $R_{3} ; 2$ translations along axes $T_{1}$ and $T_{2}$ ) and there is no direct control of the X-ray source (see figure 1(b) . Therefore, we translate the patient's table in order to compensate for the translation of the X-ray source with respect to the table. The guidance is supported by pose estimation using the attached video camera. We designed a planar marker pattern, in which all the square markers can be uniquely detected [4. The corners of each square marker can be extracted with subpixel accuracy and used as feature points. Having the marker pattern with known geometry, we are able to establish point correspondences between the $2 \mathrm{D}$ image points and $3 \mathrm{D}$ space points at the marker pattern. Based on these point correspondences, the pose of the camera (X-ray source) relative to the marker pattern (patient's table) is computed by using a camera calibration method [5]. The estimation of the pose is performed with a frequency of $8-10 \mathrm{~Hz}$ in our implementation. This can provide a continuous feedback for the translation of the table. Kainz et al. [6] developed a method to do C-arm pose estimation using an X-ray visible planar marker pattern attached to the operating table. It is not suitable as guidance for positioning of the table, since a continuous X-ray exposure and therefore a large amount of radiation is inevitable. After translation compensation, the rotation $R$ of the camera can be computed from pose estimation. $K$ was obtained during the CamC calibration. Therefore, the homography for aligning X-ray images can be computed and it is valid for all image points. To generate a parallaxfree panoramic X-ray image, the first acquired X-ray image is defined as the reference image, and all others will be registered into its coordinate system (see figure 2(b)].

Model of C-arm X-ray Imaging with Fixed Intrinsic Parameters. The $\mathrm{X}$-ray source and the detector plane are loosely coupled, mounted on the opposite sides of the C-arm. Due to gravity, it is hard to suppose a fixed relation between the $\mathrm{X}$-ray source and the detector plane, i.e. intrinsic parameters change, in different $\mathrm{C}$-arm orientations. However, the C-arm orientation must be changed to achieve a parallax-free stitching. In order to reduce the error caused by the changes of the $\mathrm{C}$-arm orientation in the final panoramas, we adapted the idea 
of the Virtual Detector Plane (VDP) proposed in [7. Four X-ray markers, attached on the back of the mirror, are roughly fixed to the $\mathrm{X}$-ray source in all C-arm orientations. A homography $H_{v i r} \in \mathbb{R}^{3 \times 3}$ is estimated by the four X-ray marker point correspondances in two X-ray images such that $m_{x}^{\text {ref }}=H_{v i r} m_{x}$, in which $m_{x}$ is the image point in the current acquired X-ray image and $m_{x}^{r e f}$ is the image point in the reference X-ray image acquired in the calibrated $\mathrm{C}$-arm orientation. We used the Normalized Direct Linear Transformation 8 to estimate the homography $H_{v i r}$. Before applying the planar homography computed in the calibration of the CamC system, the X-ray image was first warped to the virtual detector plane by $H_{v i r}$ in order to guarante fixed intrinsic parameters.

\section{Experiments and Results}

In our experiments, the image acquisition protocol was the following, after acquiring the first X-ray image, the second X-ray image was acquired by first repositioning the $\mathrm{C}$-arm with its available rotations, then performing a guided translation of the table following the feedbacks from pose estimation. Finally, the relative $\mathrm{X}$-ray source motion to the table was considered as a pure rotation if the relative translation of the X-ray source was below $1 \mathrm{~mm}$. All following X-ray images were acquired in the same way like the acquisition of the second image.

In order to quantify the accuracy of the panoramic X-ray images generated by our parallax-free X-ray image stitching method, we conducted an experiment to compute the difference of the overlapping area of two stitched X-ray images. One possibility to compute this difference is using similarity measurements. However, very limited feature information and high noise in the bone X-ray images make similarity measurements unreliable. This is the reason why others have employed the ruler [2] or video images [3] for X-ray image stitching. We constructed a phantom composed of spherical X-ray markers on three different planes (see figure 3(b) and attached it to the operating table. We performed our method to stitch two X-ray images, in the overlapping area of which at least two X-ray markers on each plane were imaged. The centroids of these markers were extracted with subpixel accuracy in the two X-ray images and used to compute the difference of the overlapping area which is the distance between corresponding centroid pairs (see figure 3(b). This procedure was performed twice. We evaluated the distances with and without applying the VDP method respectively. The overall errors were found to be $1.93 \pm 1.36$ pixels without VDP and $1.76 \pm 1.36$ pixels with VDP correction. The improvement of applying the VDP is negligible, since the X-ray source undergoes very limited rotations (around $5^{\circ}$ ).

Our parallax-free X-ray stitching was further validated in comparison to non parallax-free X-ray stitching of a cow's femoral bone. We implemented a similar method [3] for non parallax-free X-ray stitching. In the experiment, the cow bone was positioned on the operating table, and two metal tools were placed below the bone. The bone was not exactly positioned parallel to the marker pattern plane due to its big distal end. This setup is close to real clinical cases, in which it is hard to assume that a long bone is placed parallel to the marker pattern 


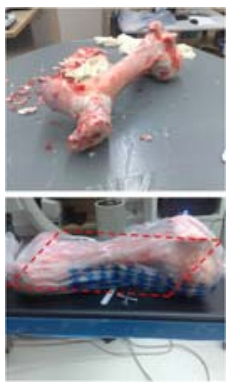

(a) Setup

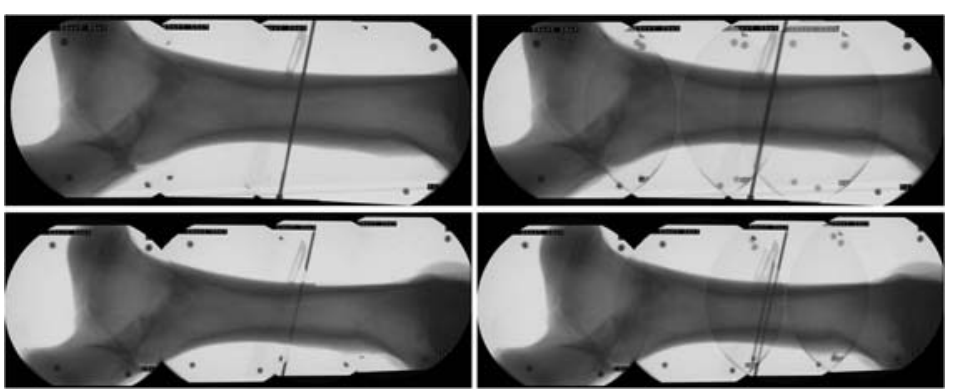

(b) Panoramic X-ray Images

Fig. 4. (a) A real cow's femoral bone and the setup for generating panoramic X-ray images; (b) The top images show the parallax-free panoramic X-ray images generated by parallax-free stitching. The bottom images show the panoramic X-ray images generated by non parallax-free stitching. In each row, the left and the right panoramas were created by using the maximum pixel values and the average pixel values respectively in the overlapping areas.

plane due to the soft tissue, muscle and fat around the bone. We created the panoramic X-ray images by using maximum pixel values and average pixel values respectively in the overlapping areas. From visual inspection, the maximum yields the most uniform image but hides the misalignments (parallax effects) in the panoramas, and the average makes the individual X-ray images visible in the panoramas. Figure 4(b) shows the resulting images. A non parallax-free panoramic X-ray image was generated using the planar transformation computed for the plane (marked by red dash lines roughly in figure 4(a) intersecting the middle of the bone horizontally and parallel to the marker pattern plane. This panoramic image has minor discontinuities in the bone boundary and clear parallax effects in the metal tools. Compared to non parallax-free stitching, parallaxfree stitching produced a true parallax-free panoramic X-ray image, in which the cow bone and metal tools are almost perfectly aligned at the same time. Note that, the overlap between the consecutive images is used to illustrate the quality of the created panoramic X-ray image, and it is not necessarily required since our stitching method is independent of X-ray images.

\section{Discussion and Conclusion}

We presented a novel method to generate parallax-free panoramic X-ray images during surgery by using the CamC system and a planar marker pattern. However, our method is not limited to using the CamC system and can be easily applied to other C-arm setups as long as the pose of the $\mathrm{C}$-arm relative to the patient's table is available, such as using an external tracking system. In our system setup, the marker pattern was attached under the operating table and is thus invisible to surgical crew during the whole operation. This allows our solution to be smoothly integrated into the surgical procedure. Our method does 
not rely on overlapping $\mathrm{X}$-ray regions and does not require a fronto-parallel Carm setup. Unlike existing methods, we are able to generate a true parallax-free panoramic X-ray image that can be treated as a single image obtained from a $\mathrm{C}$-arm having an exceptional wide imaging field of view. We additionally implemented a method to reduce the error caused by varying intrinsic parameters of $\mathrm{C}$-arm X-ray imaging. The results show that the parallax-free panoramic X-ray image generated by our method is accurate enough and has a high visual quality. Trauma surgeons confirmed that, apart from long bone surgery, it will also support a wide range of potential intra-operative X-ray imaging applications such as pelvic fracture or whole-spine imaging, since our method is independent of the configuration of bone structures and suitable for any part of body. The table motion in our experiments is mainly translational in forward-backward direction. Currently, surgeons need put additional efforts to move the table to the correct position. However, motorized tables are already available in many clinical sites, which could further support the translation of the table. We believe that our solution for creating panoramic X-ray images has a high potential to be introduced in everyday surgical routine, and will allow surgeons to validate the quality of their treatment during orthopedic and trauma surgery with minimized radiation.

Acknowledgments. Thank to Siemens Healthcare for partial financial support.

\section{References}

1. Navab, N., Mitschke, M., Bani-Hashemi, A.: Merging visible and invisible: Two camera-augmented mobile C-arm (CAMC) applications. In: Proc. IEEE and ACM Int'l Workshop on Augmented Reality, San Francisco, CA, USA, pp. 134-141 (1999)

2. Yaniv, Z., Joskowicz, L.: Long bone panoramas from fluoroscopic x-ray images. IEEE transactions on medical imaging 23(1), 26-35 (2004)

3. Wang, L., Traub, J., Heining, S.M., Benhimane, S., Euler, E., Graumann, R., Navab, N.: Long bone x-ray image stitching using camera augmented mobile c-arm. In: Metaxas, D., Axel, L., Fichtinger, G., Székely, G. (eds.) MICCAI 2008, Part II. LNCS, vol. 5242, pp. 578-586. Springer, Heidelberg (2008)

4. Zhang, X., Fronz, S., Navab, N.: Visual marker detection and decoding in ar systems: A comparative study. In: IEEE International Symposium on Mixed and Augmented Reality (ISMAR 2002) (October 2002)

5. Zhang, Z.: A flexible new technique for camera calibration. Technical report, Microsoft Research (1998) (last updated in 2008)

6. Kainz, B., Grabner, M., Rüther, M.: Fast marker based c-arm pose estimation. In: Metaxas, D., Axel, L., Fichtinger, G., Székely, G. (eds.) MICCAI 2008, Part II. LNCS, vol. 5242, pp. 652-659. Springer, Heidelberg (2008)

7. Navab, N., Mitschke, M.: Method and apparatus using a virtual detector for threedimensional reconstruction form x-ray images (2001); Patent US 6236704; Filing date: June 30, 1999; Issue date: May 22

8. Hartley, R., Zisserman, A.: Multiple View Geometry in Computer Vision. Cambridge University Press, New York (2003) 